\title{
Percepção de pessoas idosas sobre a influência da espiritualidade em sua saúde e qualidade de vida
}

\author{
Perception of elderly people on the influence of spirituality their health and quality of life \\ Percepción de las personas mayores sobre la influencia de la espiritualidad su salud y \\ calidad de vida
}

Luana da Cruz da Silva1 ${ }^{1}$, Mariana da Silva de Jesus Marques ${ }^{1}$, Luna Vitória Cajé Moura ${ }^{1}$, Rosangela Jeniffer Soares Rosa ${ }^{1 *}$, Anne Eugênia Ledo Gonçalves Viana ${ }^{1}$, Jordelma Medeiros Lima1, Aldones Adenilton Sousa Ribeiro¹.

\section{RESUMO}

Objetivo: Conhecer a percepção de pessoas idosas sobre a influência da espiritualidade, sua saúde e qualidade de vida. Métodos: Trata-se de uma pesquisa exploratória, descritiva, de abordagem qualitativa. Foram entrevistados doze idosos que frequentavam um Grupo da Terceira Idade na zona rual de um Município do Recôncavo baiano, no ano de 2020. A coleta de dados foram utilizados um questionário e um roteiro de entrevista semiestruturado. Os dados analisados com a utilização da técnica de análise temática de conteúdo de Bardin L. (2011). Resultados: Houve um índice favorável quanto a percepção desses indivíduos sobre aespiritualidade e a influência em sua saúde e qualidade de vida. É relevante ressaltar o quanto os mesmos aplicam essas práticas em suas vidas diárias como: orar, estudar artigos religiosos e dentre outras para ter comunhão com seu criador (Deus). Observou-se que esses idosos são bastante confiantes ao expressar sobre a importância de manter a confiança em uma divindade que os auxiliam em todos os momentos. Conclusão: A espiritualidade desempenha um papel apreciável na vivência desses indivíduos, concedendo uma perspectiva de segurança, para enfrentar as dificuldades do dia a dia e alento para os processos fisiológicos e patológicos que os acometem na terceira idade.

Palavras-chave: Idoso, Saúde e espiritualidade, Qualidade de vida.

\begin{abstract}
Objective: The study aimed to illuminate the old people's perception about the influence of spirituality in their health and quality of life. Methods: It is an exploratory, descriptive research, with a qualitative approach. Twelve elderly people who attended a Group of the Elderly were interviewed in the rural area of a municipality in the Recôncavo of Bahia, in the year 2020. The data collection used a questionnaire and a semi-structured interview script. The data analyzed using the technique of thematic content analysis by Bardin L. (2011). Results: The study proved a favorable index regarding the perception of these individuals about spirituality and the influence on their health and quality of life. Daily's practices as: praying, studying religious articles and among others to have fellowship with their creator (God), was too relevant for their lifes. Observed quite confident when expressing about the importance of maintaining trust in a deity who help them all the time. Conclusion: Spirituality plays an appreciable role in the experience of these individuals, providing a perspective of security, to face the issues of normal life and encouragement for the physiological and pathological processes that affect them in old age.
\end{abstract}

Keywords: Elderly, Health and spirituality, Quality of life.

${ }_{1}^{1}$ Faculdade Adventista da Bahia (FADBA), Cachoeira - BA. *E-mail: rosangela_jeniffer@hotmail.com

SUBMETIDO EM: 5/2021 | ACEITO EM: 5/2021 | PUBLICADO EM: 6/2021 


\section{RESUMEN}

Objetivo: Conocer la percepción de las personas mayores sobre la influencia de la espiritualidade, su salud y calidad de vida. Métodos: Se trata de una investigación exploratoria, descriptiva, con enfoque cualitativo. Doce ancianos que asistieron a un Grupo de Ancianos fueron entrevistados en el área rural de un municipio del Recóncavo de Bahía, en el año 2020. La recolección de datos utilizó un cuestionario y un guión de entrevista semiestructurado. Los datos analizados mediante la técnica de análisis de contenido temático de Bardin L. (2011). Para la recolección de datos se utilizó un cuestionario y un guión de entrevista semiestructurado. Los datos se analizaron mediante la técnica de análisis de contenido temático de Bardin. Resultados: Hubo un índice favorable en cuanto a la percepción de estos individuos sobre espiritualidad y influencia en su salud y calidad de vida. Es relevante enfatizar cuánto aplican estas prácticas en su cotidiano: orar, estudiar artículos religiosos y entre otros, tener comunión con creador. Se observó que los adultos mayores, se presentan bastante confiados al expresar la importancia de mantener la confianza en una deidad que los ayude en todo momento. Conclusión: La espiritualidad es crucial en la experiencia de estos individuos, otorgando una perspectiva de seguridad para enfrentar las dificultades de la vida y el estímulo de los procesos fisiológicos y patológicos que los afectan en la vejez.

Palabras clave: Anciano, Salud y espiritualidad, Calidad de vida.

\section{INTRODUÇÃO}

Sabe-se que hoje a população vem envelhecendo de modo exponencial, e este envelhecimento vem acompanhado de doenças crônicas não transmissíveis em substituição às infectocontagiosas que se mostravam prevalente em tempos atrás. Por se tratar de doenças crônicas, existem vários fatores que contribuem para que os idosos tenham uma estimativa de vida mais longa (ORGANIZAÇÃO MUNDIAL DA SAÚDE, 2015).

Devido todo esse enfrentamento que os idosos passam, sejam elas doenças, perda familiar, e de sua vida social que trazem tristezas e isolamentos, é importante entender o significado da espiritualidade na vida dos idosos, e assim compreender que os mesmos se agarram a um ser supremo que os criou e que proporciona uma sensação de paz e bem-estar, aliviando as consequências que os acometem. A dimensão da espiritualidade é igualmente relevante quando associada à esperança, trazendo novos significados para a vida, sobretudo em tempos de dificuldades e adversidades (SOUZA EN, et al. (2017).

Conforme Balbinotti HB (2017), através da espiritualidade, á esperança e as forças renascem na vida das pessoas, pois elas tendem á acreditar que tudo tem um porquê, um ser ou força superior os ampara nas adversidades. Com todas as expectativas que a envolvem, é um fator que ajuda alcançar uma qualidade de vida e saúde, por motivar a enfrentar os desafios e consequências, tendo um desenvolvimento melhor com a sociedade, compartilhando com aos outros a sua fé, esperança e estilo de vida, fortalecendo uns aos outros com novas percepções sobre a espiritualidade, sua influência e relevância.

Para Nunes MG, et al.(2017) sobre o conceito de espiritualidade ele afirma que a mesma não está necessariamente vinculada a uma crença em Deus; remete a uma questão universal e está baseada em um propósito de vida.

Portanto, após o exposto, o objetivo deste estudo foi conhecer a percepção de pessoas idosas sobre a influência daespiritualidade em sua saúde e qualidade de vida.

\section{MÉTODOS}

Trata-se de uma pesquisa exploratória e descritiva de abordagem qualitativa. A pesquisa envolveu um grupo social (de cunho religioso) que implementa a prática do envelhecimento ativo em uma comunidade, na zona rural de um município do recôncavo da Bahia, no ano de 2020.

Participaram desse estudo doze idosos de acordo com os seguintes critérios de inclusão: idosos que explicitam voluntariedade na participação com assinatura do Termo de Consentimento Livre e Esclarecido (TCLE), ter no mínimo 60 (sessenta) anos, ter condições de responder aos instrumentos de autorrelato, e participar ativamente do grupo de reuniões da referida comunidade. 
Destaca-se que o estudo foi realizado considerando-se as observâncias éticas contempladas na Resolução 466/12 da Comissão Nacional de Saúde, sendo o projeto de pesquisa aprovado pelo Comitê de Ética em Pesquisa da Universidade Federal da Bahia (UFBA), conforme parecer de CAAE ำ33500720.3.0000.5543.

A coleta de dados ocorreu na residência dos idosos em meados de setembro e outubro de 2020, foram utilizados um questionário e um roteiro de entrevista semiestruturado. É importante salientar que foram tomadas todas as medidas cabíveis de proteção para realização da coleta de dados, respeitando o distanciamento, a utilização de equipamentos de proteção individual (EPI), tais como máscara, luvas e touca; e até mesmo evitando adentrar ademais cômodos da casa do participante. Tudo para garantir a segurança dos idosos e seus familiares bem como a das pesquisadoras.

Para a coleta de dados foi utilizado inicialmente o Instrumento de Qualidade de Vida da Organização Mundial da Saúde - Módula Espiritualidade, Religiosidade e Crenças Pessoais (WHOQOL-SRPB, em inglês), é um módulo adicional do WHOQOL para avaliar espiritualidade, religião e crenças pessoais como componentes da estrutura da qualidade de vida. Esse instrumento é composto de 32 itens divididos em oito fatores (Conexão a Ser ou Força Espiritual, Sentido na Vida, Admiração, Totalidade e Integração, Força Espiritual, Paz Interior, Esperança e Otimismo, e Fé) num índice geral composto por quatro itens (SRPB Global) originalmente pertencentes ao domínio SRPB do WHOQOL-100, Panzini RG (2011).

A coleta contou também com o auxílio de um roteiro semiestruturado para a entrevista, que foi elaborado para este fim, contendo perguntas norteadoras e pertinentes que dizem respeito a espiritualidade do idoso e a influência que a mesma pode exercer na saúde e na qualidade de vida dessas pessoas idosas.

As entrevistas foram gravadas em áudio com o auxílio do celular e foram transcritas posteriormente. 0 discurso emergido através das entrevistas individuais sobre a espiritualidade dos idosos, foi analisado com a utilização da técnica da Análise temática de conteúdo (BARDIN L, 2011). A intenção da utilização desta técnica foi fazer esta coletividade de idosos falarem, nos resultados do estudo, respondendo às questões do estudo por meio das perguntas norteadoras aplicadas individualmente. Deste modo se fez conhecida a percepção de pessoas idosas sobre a influência da espiritualidade em sua saúde e qualidade de vida.

\section{RESULTADOS E DISCUSSÃO}

Para melhor compreensão sobre a percepção dos idosos acerca da influência da espiritualidade em sua saúde, a partir das falas, foram elaboradas quatro categorias e seus delineamentos: percepção dos idosos acerca do que é espiritualidade; práticas espirituais desenvolvidas pelos idosos; percepção sobre a influência da espiritualidade em sua saúde; e percepção sobre a influência da espiritualidade em sua qualidade de vida como apresentadas a seguir:

\section{Percepção dos idosos acerca do que é espiritualidade}

Segundo o estudo de Chaves LJ e Gil CA (2015) quanto a concepção da espiritualidade, foram destacados com maior número de referência para os idosos a relação com o Sagrado, Apoio e Transcedência, nesse estudo a metade dos entrevistados consideram a Espiritualidade como fonte de apoio, é vista como forma de sustentação fundamental para todas as áreas da vida, no caso dos pacientes ancológicos acreditar e se apoiar em Deus favorece no proagnóstico logo, o viver fica mais seguro e tranquilo. Para os idosos a percepção de espiritualidade é bem satisfatória, indicando bem-estar, segundo um dos idosos entrevistado o ser humano possui um vazio e este vazio só pode ser preenchido por Deus.

Todos os entrevistados consideraram a Espiritualidade como comunhão com Deus:

"É buscar Deus cada manhã, é meditar, é orar" (Idosa 9).

"Espiritualidade para mim é comunhão com Deus, a ligação com Deus é básica como o alimento; a comida, água, você não pode sobreviver sem ela, assim para mim é minha espiritualidade... eu sou dependente, carente de ti [Deus], sem ti eu não posso viver" (Idosa 3). 
O estudo de Scortegagna HM, et al. (2018), trouxe resultado semelhante quando todos osentrevistados da sua pesquisa afirmaram ter religião e vivenciar a sua espiritualidade/religiosidade atribuindoesse vivenciar a estar em comunhão com o seu Ser Superior. Outro estudo que avaliou também a definição de espiritualidade envolviam também as ideias de conexão com poder divino (GUTZ L e CAMARGO BV, 2013).

\section{Desempenhar práticas espirituais e religiosas}

Diante das respostas dos idosos na entrevista, houve uma compreensão do que realmente eles expressam sobre a comunhão com Deus, e os benefícios que a leitura da Palavra (Bíblia), traz em suas vidas.

\section{"[...] É a comunhão com Deus e você praticar né? É realmente uma comunhão com Deus de estudo da Palavra" (Idosa 4).}

Quanto ao desempenho das práticas espirituais e religiosas efetivada pelos idosos, outros estudos validamos resultados encontrados pontuando que as práticas de ler o Livro Sagrado de alguma religião, retirar-se para um local isolado para oração são práticas realizadas pela maioria dos idosos entrevistados e a participação em atividades da igreja (GUTZ L e CAMARGO BV, 2013; SCORTEGAGNA HM, et al., 2018).

Ao abordar os assuntos sobre a espiritualidade com os idosos, foi notório o quanto acreditam que o bom relacionamento com o próximo não vem de si mesmos, e sim de um ser superior. Diante disso é interessante entender o quanto a espiritualidade influencia na qualidade de vida desses indivíduos.

"[...] Espiritualidade é a qualidade da gente abraçar o nosso próximo, [...]. A espiritualidade está voltada também para o nosso relacionamento com Deus, maisdo que tudo no mundo. E o nosso envolvimento para com o próximo é justamente fruto do nosso relacionamento com Deus [...]" (Idosa 11).

O estudo de Pallini AC, et al. (2019) confirma ao trazer resultado semelhante onde a maioria dos participantes da pesquisa relataram que ao estarem envolvidos em reuniões da igreja, orações, dedicarem tempo para se conectarem com Deus, fazia com que eles exercessem mais paciência e a vontade de querer estar perto de familiares e amigos.

\section{Práticas espirituais desenvolvidas pelos idosos}

Segundo os idosos, existem várias práticas que eles desenvolvem no dia a dia, como: reunir em grupos para orar, bem como suas orações pessoais; meditação, estudo da Bíblia sobre temas relacionados às doutrinas de sua crença; leituras de livros religiosos e também realizam culto familiar.

\section{Oração}

Na percepção sobre a oração, os idosos relataram ser o primeiro elo para que se tenha uma boa comunhão e uma comunicação com o ser divino. Além do ato de orar, acreditam que é fundamental falar dessa divindade para outras pessoas, o quanto elas necessitam acreditar que existe um criador.

"Oração! Em primeiro lugar, ela é a nossa comunhão com Deus, ligação direta com o nosso Criador, não é isso? Falar de Deus para os outros, principalmente para aqueles que ainda não conhecem a Deus [...]" (Idosa 11).

Outros estudos também referiram a oração como sendo a principal prática privada realizada pelos idosos, quer seja na privacidade do lar, em uma Instituição de Longa permanência de Idosos (ILPI), ou como prática pública em participação de encontros religiosos (GUTZ L e CAMARGO BV, 2013; SCORTEGAGNA HM, et al., 2018).

\section{Leitura de publicações religiosas}

Outras práticas espirituais relatadas pelos participantes idosos incluíram: meditações diárias, leitura da Bíblia, livros religiosos, e participar de cultos.

"Então através da espiritualidade e comunhão com Deus, eu pratico exercícios espirituais, eu leio o espírito de profecia de Ellen White, mulher inspirada por Deus, faço leitura da Bíblia, e realizo os cultos pela manhã e à tarde segundo a orientaçãoda igreja e que a Bíblia dá" (Idoso 7). 


\section{Consumo de material religioso em meios digitais}

Outra prática apresentada pelos idosos para desempenhar sua espiritualidade, foi à utilização de meios digitais. Percebe-se que a inclusão digital está em crescimento entre a população idosa. Relataram que usam os meios digitais televisão e celular para assistir programas religiosos, ouvir música, enviar mensagens para os outros e formar grupos de oração. Ressaltam que foi, e ainda continua sendo uma ferramenta que os auxilia a superar os desafios que a pandemia da COVID-19 trouxe com isolamento social.

"[...] Outras formas "é" a leitura da Bíblia, a leitura da lição, ir à igreja, a pregação desses pastores na televisão. Ir à igreja é muito bom, mas essa forma de a gente em casa assistir essas pregações, esses vídeos, isso foi realmente maravilhoso, néajuda muito a gente, a gente fica esperando chegar o sábado pra gente poder assistir" (Idosa 1).

Quanto ao consumo de material religioso em meios digitais obteve-se resultado condizente ao encontrado em outro estudo associando que quanto maior fosse à idade maior seria era o consumo de programas religiosos televisionados (PALLINI AC, et al., 2019).

\section{Separação de tempo no dia para contato com o ser superior}

A espiritualidade é uma prática exclusiva dos seres humanos e, em pessoas idosas, ela tem se demonstrado mais presente (COSTA FB e TERRA NL, 2013).

"Primeiro eu tenho meu momento pessoal, que nós devemos ter né! E eu busco desenvolver principalmente depois que eu tenho meu momento espiritual com Deus,eu busco desenvolver principalmente dentro do meu lar, testemunho!" (Idosa 5).

"[...] acordo de madrugada pedindo a Jesus que me dê força, coragem, ânimo, [...]. (Idosa 12).

Esse resultado é semelhante ao encontrado em outro estudo em que os participantes utilizavam a espiritualidade no dia a dia, em que os mesmos referiam manter um relacionamento diário em que trazia esperança e dava força (PALLINI AC, et al., 2019).

\section{Percepção sobre a influência da espiritualidade em sua saúde:}

\section{Influência na saúde mental}

Eles expressam muito o quanto essa influência de ter um grupo que os apoia, em uma comunidade ligada a espiritualidade é significante, onde auxiliam as pessoas que estão passando por algum problema, ajudando em oração e visitas nos lares, proporcionando ali uma boa recuperação e superação dessas patologias ou outras adversidades da vida.

\footnotetext{
"Já, várias vezes, porque as vezes a gente tá tão ansioso com problemas não sabe resolver, aí você entra em comunhão com Deus, lê alguma coisa a respeito confia e as coisas melhoram [...]" (Idosa 8).
}

Em relação a influência da espiritualidade na saúde mental dos idosos para um envelhecimento bem sucedido, os resultados foram vistos de forma positiva onde a espiritualidade pode ser vivenciada por meio de uma conexão com o ser superior bem como com próximo (THAUVOYE E, et al., 2018; SCORTEGAGNA HM, 2018).

\section{Influência na saúde física}

Os idosos abordaram muito sobre a questão de como a influência da espiritualidade tem os ajudado a enfrentar vários problemas, dizem também que a comunhão, a confiança em Deus é essencial para superar qualquer coisa que tendem a enfrentar, reforçam que a mudança no estilo de vida é um resultado da comunhão com Deus.

"[...] Se não fosse a espiritualidade, eu não ia cuidar da minha alimentação, iria comer de qualquer forma então tudo isso ajuda" (Idosa 8). 
Através do depoimento de alguns idosos percebe-se a influência da espiritualidade/religiosidade contribuindo para a algumas práticas de bons hábitos. Essa não seria uma atitude isolada desse grupo, pois de acordo com o estudo de Koenig HG, et al. (2004). Desse modo sabemos que os hábitos e práticas alimentares são de suma importância para o desenvolvimento do corpo de forma saudável, diante disso, é o ponto central da maioria das religiões mundiais, podemos citar: o jejum, aprovação de alguns alimentos e proibições de outros (SEIXAS MC, 2017).

\section{Contribui para um enfrentamento positivo no luto}

É muito interessante essas reflexões que os idosos apresentam e descrevem sobre essa ligação, e comunhão a um ser superior, no qual os ajudam e sempre está presente dando forças e resiliência para superar qualquer conflito.

"[...] Eu morro de medo de cirurgia, eu já fiz sete, cada cirurgia marcada pra mim é um impacto. Essa última, foi uma surpresa maior do mundo, eu não esperava nunca, mas graças a Deus eu "sobressai", porque Deus me ajudou" (Idosa 5).

No questionário Instrumento de Qualidade de Vida da Organização Mundial da Saúde - Módula Espiritualidade, Religiosidade e Crenças Pessoais (WHOQOL-SRPB, em inglês) que foi aplicado aos idosos durante a entrevista, essas respostas se repetem também quando a maioria apresenta escores entre quatro e cinco nos critérios "resiliência em situações difíceis" e "esperança".

Isso é visto de forma positiva em sua vida, pois minimiza os riscos de depressão vivenciada por tantos nessa idade, conforme Duarte FM e Wanderley KF (2011) afirmam nos resultados do seu estudo onde sintomas depressivos foram menos frequentes nos que eram mais religiosos. Dados equivalentes a esta pesquisa foram encontrados em outro estudo realizado sobre o papel do suporte social na associação entre religiosidade e saúde mental em idosos de baixa renda, foram identificados fortes níveis de associação entre frequência religiosa com a menor prevalência de transtornos mentais comuns e melhoria da saúde física (CORRÊA AA, et al., 2011).

\section{Percepção sobre a influência da espiritualidade em sua qualidade de vida}

Através dos relatos se pôde nomear benefícios como: contribuição para as relações interpessoais, formação de uma rede de apoio social e emocional, promoção de sentimento de segurança e conforto, promoção da resiliência em situações desafiadoras.

\section{Relações interpessoais}

Os idosos participantes atribuem uma melhor relação interpessoal à espiritualidade, dentre elas a compreender e se relacionar com as pessoas, principalmente com os seus familiares.

"[...] A espiritualidade, ela sempre fez toda a diferença na minha vida, [...] aprendi na Sua Palavra, cada dia eu aprendia uma coisa, a compreender as pessoas. Atravésda espiritualidade a gente aprende como lidar com as pessoas, como lidar com o esposo, como lidar com a minha irmã que está com depressão e poder ajudar ela" (Idosa 6).

De modo semelhante em outro estudo os participantes demonstraram-se satisfeitos com o relacionamento interpessoal, pois os vínculos sociais na percepção deles influenciam na qualidade de vida e tem se mostrado favorável na velhice (OLIVEIRA LS, et al., 2012).

\section{Apresenta uma rede de apoio social e emocional}

É possível notar que metade da amostra deste estudo é composta por idosas viúvas, o que denota um possível grau de solidão. Outro fator característico dessas mulheres é que a maioria delas mora sozinha.

Outra característica em comum deles é a de que ele migram de outros estados ou cidades para morar na referida localidade, fenômeno comum no bairro que se configura como bairro majoritariamente de estudantes e familiares, mas o diferencial na vida desses idosos, é que foram há bastante tempo, quando seus filhos 
ainda eram jovens, com o intuito de os ofertar uma educação melhor baseada na sua. Então, os filhos foram sendo formados, e por se tratar de um lugar agradável e tranquilo para o desenvolvimento de sua fé, muitos permaneceram. Por essa razão moram sós sem seus familiares.

"[...] vou pro culto, vou pro pequeno grupo da terceira idade, temos os cultos nas casas, [...] temos pequeno grupo, aí eu vou e me sinto bem a cabeça parece que esvazia, durmo bem, e passo dois ou três dias bem mesmo 'eu tô orando por você' aí isso nos fortalece a gente, porque é boa a gente saber que tem alguém orando pela gente, imagine um grupo? E quando eu vou ao grupo da terceira idade, aí muitos falam 'orei por você"' (Idosa 2).

Em um estudo em que investigava a percepção de idosos acerca dos grupos de convivência na cidade de Cajazeiras-PB trouxe resultado semelhante quando questionados sobre os motivos que o levavam a participar desses grupos de convivência as respostas identificadas foram: solidão e a necessidade de atividades de lazer (ANDRADE NA, et al., 2014).

\section{Promove sentimento de segurança e conforto}

Uma das essências da religião ou espiritualidade é proporcionar uma sensação de segurança e conforto, e isso foi comprovado através dos relatos da maioria dos idosos:

"Se obtém muita coisa. Uma dessas são respostas que a gente jamais pensaria que teria. , então eu agradeço muito a Deus pela companhia e por sempre me ouvir" (Idosa 9).

O estudo de Chaves LJ e Gil CA (2015) corrobora com tais achados, através das falas das idosas a seguir:

"[...] se a gente não tem um apoio espiritual [...] a vida está tão difícil [. ] a violência está desgraçando [...]. Me parece que a vida se tornou muito banal [...]. Então a gente tem que ter um apoio espiritual [...]" (Idoso 10).

O estudo de Duarte FM e Wanderley KF (2011) confirma tais achados quando aponta que a religião ou espiritualidade apresentam uma relação de otimismo, esperança de melhora, no cultivo de força em um ser superior que possa auxiliar.

\section{Promove bem-estar e sentido para a vida}

Ao entrevistar os idosos, os mesmos expressaram que através da crença e das práticas religiosas podem obter várias coisas no âmbito de suas vidas, acreditam que sua existência, sua vida. Tende a entender que se não fosse essa fé em um ser divino a vida não existiria e não saberiam como viver.

Outro aspecto importante que podemos observar na entrevista com esses idosos, foi sobre a preocupação em manter um estilo de vida mais saudável, que também é motivado pela sua espiritualidade.

"A gente pode obter tudo, principalmente a vida! A vida que se obtém através do encontro com o Salvador" (Idosa 6).

Da mesma forma, um estudo demonstrou que a vivência da espiritualidade/religiosidade por parte dos idosos se revelou como uma fonte de bem-estar para lidar com as adversidades que a vida apresenta isso foi visto de forma positiva (SCORTEGAGNA HM, et al., 2018).

No questionário que foi aplicado essas respostas também se confirmam quando a maioria apresenta novamente escores entre quatro e cinco (significando "bastante" e "extremamente") acerca do critério de "bem-estar" e "sentido para vida", se referindo aos impactos da espiritualidade.

Pode-se inferir que, de acordo com os depoimentos apresentados pelos idosos no estudo de Reis LA e Menezes TM (2017), a espiritualidade ocupa uma posição central na vida deles; e que a prática de atividades religiosas e espirituais, como: leitura da Bíblia, rezar o terço e orar, são estratégias que fazem parte da rotina para o enfretamento de situações adversas, auxiliado na recuperação, manutenção e prevenção da saúde, proteção pessoal e familiar e, contribui para um envelhecimento sereno. 


\section{CONCLUSÃO}

O estudo permitiu uma ampla reflexão sobre a percepção relacionada a espiritualidade na saúde e qualidade de vida das pessoas idosas, e a maneira de como elas desenvolvem suas vidas. Dentro disso fazse necessário que os profissionais de saúde se engajem ainda mais nas buscas de aprimorar os conhecimentos dessas práticas, para que saibam abordar de forma eficiente nas consultas realizadas, respeitando suas escolhas. Logo, conclui-se que há uma necessidade de incentivar ações que estimulem as pessoas idosas a desenvolverem ainda mais espiritualmente, visto que contribui para melhores índices de qualidade de vida, hábitos mais saudáveis e resiliência nos problemas de saúde.

\section{REFERÊNCIAS}

1. ANDRADE NA, et al. Percepção de idosos sobre grupo de convivência: estudo na cidade de Cajazeiras-PB. Rev. Bras. Geriatr. Gerontol., Rio de Janeiro, 2014; 17(1): 39-48.

2. BALBINOTTI HB. A importância da espiritualidade no envelhecimento. Memorialidades, Porto Alegre, $2017 ;$ (27 e 28): $13-44$

3. BARDIN L. Análise de conteúdo. Trad. Luís A e Augusto P. 5. Ed. Lisboa: edições 70 LTDA, 2011.

4. CAVALLI AS, et al. Motivação de pessoas idosas para a prática de atividade física: estudo comparativo entre dois programas universitários - Brasil e Portugal. Revista Brasileira de Geriatria e Gerontologia, Rio de Janeiro, 2014; 17(2) : 255-264.

5. CHAVES LJ, GIL CA. Concepções de idosos sobre espiritualidade relacionada ao envelhecimento e qualidade devida. Ciênc. saúde coletiva, Rio de Janeiro,2015; 20(12): 3641-3652.

6. COSTA FB, TERRA NL. Espiritualidade, religiosidade e qualidade de vida em idosos. Revista Geriatria e Gerontologia, Rio de Janeiro, 2013; 7(3):173-178.

7. DIAS EN, PAIS-RIBEIRO JL. Espiritualidade e qualidade de vida de pessoas idosas: um estudo relacional. Psic., Saúde \& Doenças, Lisboa, 2018; 19(3): 591-604.

8. DUARTE FM, WANDERLEY KF. Religião e espiritualidade de idosos internados em uma enfermaria geriátrica. Psicologia: Teoria e Pesquisa, 2011; 27 (1): 49 -53.

9. GLIDDEN RF, et al. A participação de idosos em grupos de terceira idade e sua relação com satisfação com suporte social e otimismo. Bol. - Acad. Paul. Psicol., São Paulo, 2019; 39 (97):261-275.

10. GUTZ L, CAMARGO BV. Espiritualidade entre idosos mais velhos: um estudo de representações sociais. Revista Brasileira de Geriatria e Gerontologia, Rio de Janeiro, 2013;16 (4): 793-804.

11. KOENIG HG, et al. Religion, Spirituality, and Health in Medically III Hospitalized Older Patients. J Am Geriatrics Society 2004; 52(4): 554-562.

12. MELO CF, et al. Correlação entre religiosidade, espiritualidade e qualidade de vida: uma revisão de literatura. Estud. pesqui. Psicol, Rio de Janeiro, 2015; 15(2): 447-464.

13. NUNES MG, et al. Idosos longevos: avaliação da qualidade de vida no domínio da espiritualidade, da religiosidadee de crenças pessoais. Saúde debate, Rio de Janeiro, 2017; 41(115):1102-1115.

14. OLIVEIRA LS, et al. Estilo de vida, senso de controle e qualidade de vida: um estudo com a população idosa de Patos de Minas-MG. Estudos e Pesquisas em Psicologia, 2012; 12(2).

15. ORGANIZAÇÃO MUNDIAL DA SAÚDE (OMS). Resumo relatório mundial de envelhecimento e saúde 2015.

16. PALLINI AC, et al. Percepções de pacientes oncológicos sobre espiritualidade: um estudo qualitativo. Psicol. Am. Lat., México, 2019; (32): 169-179.

17. PANZINI RG, et al. Validação brasileira do Instrumento de Qualidade de Vida/espiritualidade, religião e crenças pessoais. Rev. Saúde Pública, São Paulo, 2011; 45(1):153-165.

18. REIS LA, MENEZES TM. Religiosity and spirituality as resilience strategies among long-living older adults in their daily lives. Rev. Bras. Enferm.,2017; 70(4):761-6.

19. SCORTEGAGNA HM, et al. The experience of spirituality among institutionalized elderlypeople. Rev. Bras. Geriatr. Gerontol., Rio de Janeiro, 2018; $21(3)$ : 293-300.

20. SEIXAS MC. Espiritualidade no contexto da saúde. Revista Unitas, 2017; 5(2).

21. SOUZA EM, et al. Relação entre a esperança e a espiritualidade de idosos cuidadores. Rev. Texto e Contexto Enfermagem, Florianópolis, 2017; 26 (3): 67-77.

22. THAUVOYE E, et al. Spirituality and well-being in old age: exploring the dimensions of spirituality in relation to late-life functioning. J Relig Health, 2018; 57 (6): 2167-2181.

23. VEY AP, et al. Perfil das idosas participantes de um grupo de convivência. Fisioterapia Brasil, $2019 ; 20$ (1):27 - 35.

24. WICHMANN FM. Grupos de convivência como suporte ao idoso na melhoria da saúde. Rev. Bras. Geriatr. Gerontol., Rio de Janeiro, 2013; 16 (4): 821-832. 